\title{
Der Begriff „Autonomie“ in den pädagogischen Strömungen und sein geschichtlicher Hintergrund im Fremdsprachenunterricht *
}

\author{
Yunus Emre Sarı (iD), Istanbul - Gönül Karasu (D), Eskişehir \\ https://dx.doi.org/10.37583/diyalog.845601
}

\begin{abstract}
Deutsch)
Lernerautonomie wird heute als ein wichtiges pädagogisches Konzept im Rahmen der Planung des Bildungsprozesses angesehen und scheint einer der wichtigsten Voraussetzungen für die Förderung des Paradigmas des lebenslangen Lernens zu sein. Zahlreiche Autoren kommen zu einer unterschiedlichen Definition des Begriffs „Autonomie“. Dieser uneinheitliche terminologische Gebrauch ist eine Folge der etymologischen Geschichte des Begriffs „Autonomie“. Es wurde durch Literaturrecherche festgestellt, dass der konstruktivistische Ansatz, der aus der Sicht von Forschern wie u. a. Dewey, Piaget, Freire und Wygotski dargelegt wurde, eine aktive Rolle bei der Entstehung der Lernerautonomie spielte. Die Tatsache, dass der Begriff „Autonomie“ seinen Platz in vielen Strömungen und bei mehreren Autoren findet, hat zu einer Erweiterung der Bedeutung dieses Begriffes geführt. Obwohl diese Bedeutungserweiterungen das Konzept schwer verständlich machen, kann man davon sprechen, dass es sich dabei um einen wirklichen Reichtum handelt. Die Geschichte des Begriffs „Autonomie“, bevor er beim Fremdsprachenunterricht zur Anwendung kam, wird als Leitfaden dafür dienen, wie er in diesem Bereich verwendet werden sollte. Aus diesem Grund untersucht die vorliegende Arbeit das Konzept der „Autonomie“ in pädagogischen Strömungen, anhand einiger Vorläufer des Autonomiebegriffs. Dabei wird auch die Frühgeschichte der Autonomie in Bezug auf den Fremdsprachenunterricht zur Debatte gestellt.
\end{abstract}

Schlüsselwörter: Autonomie, Sprachunterricht, pädagogische Strömungen, J. J. Rousseau, J. Dewey, P. Freire.

\section{Abstract (English)}

The concept of "autonomy" in different pedagogical paradigms and its historical background in foreign language teaching

Learner autonomy is today considered an important pedagogical concept in the planning of educational processes and seems to be one of the most important prerequisites for promoting lifelong learning. Several scholars have proposed different definitions of the term "autonomy". This variant terminological use is a consequence of the etymological history of the term "autonomy". The literature suggests that the constructivist approach, which is presented from the perspectives of researchers such as Dewey, Piaget,

Einsendedatum: 29.10 .2020

Freigabe zur Veröffentlichung: 31.12.2020

* Dieser Artikel wurde aus der Doktorarbeit mit dem Titel „Feststellung der Bereitschaft der DaFLehramtsstudierenden zur Lernerautonomie: Eine Studie zur Entwicklung und Anwendung einer Skala (2020)“ von Yunus Emre Sarı unter der Leitung von Ass. Doz. Dr. Gönül KARASU erstellt. 
Freire and Vygotsky among many others, played an active role in the emergence of learner autonomy as a separate research field. The fact that the term "autonomy" finds its place in many paradigms extends the understanding of the term. The history of the term "autonomy" before it was used in foreign language teaching will serve as a guide to how it should be used in it. For this reason, the present work examines the concept of "autonomy" in different pedagogical paradigms on the basis of some prerequisites of the concept of autonomy. Also, the early history of autonomy in relation to foreign language teaching is discussed.

Keywords: Autonomy, Language teaching, pedagogical paradigms, J. J. Rousseau, J. Dewey, P. Freire. 


\section{EXTENDED ABSTRACT}

Due to the fact that the term "autonomy" has different meanings at its origin and is used in different fields (such as psychology, philosophy, politics, etc.), it has caused some differences of meaning in the foreign language field.

In order to better understand the meaning of "autonomy" in foreign language learning, it is important to know how the term "autonomy" came into use and why it is so important today. This article therefore deals with the historical development and the meaning of the word "autonomy".

In the first part, the concept of "autonomy" was discussed in relation to pedagogical paradigms by seminal researchers and their conceptions of the idea of autonomy such as "Jean-Jacque Rousseau", "John Dewey", "William Kilpatrick" and "Paulo Freire", as they had a huge influence on the current concept of "learner autonomy".

Constructivist approaches assume that learning is a constructive process and claim that each learner learns from his or her own "experiences" by using his or her own values, beliefs and past experiences. It can then be assumed that all these personal experiences and knowledge have matured learners in terms of both moral and learning skills.

Learning in language classes is also based on experience and knowledge. As in today's modern understanding of foreign language learning, this means that learners learn through experience and learning by doing, not by memorizing. Teaching methods that better take into account the diversity of learners in terms of intellectual abilities and learning potential are also needed. Such teaching methods require differentiated classrooms, action-oriented teaching, task-oriented learning, open teaching, station work, project work, research-developing teaching and so on. Recent studies have also shown that such learning methods have positive and better results in learning a foreign language and improve both cognitive and affective skills of learners.

However, such learning methods cannot take place in an educational model in which teachers are authoritative figures, in which knowledge and skills are taught to learners according to a prescribed curriculum. This relationship is an important factor in recognizing how close the concept of autonomy between the learners and the teachers is.

After all, if all the equipment and materials that the teacher is supposed to use in the classroom are determined in advance by an inflexible model and by official institutions, both teachers and learners are faced with a restricted learning environment.

On this basis, it is clear that the lack of autonomy of the learning group will be greater the more heteronomy ${ }^{1}$ enters the classroom. From this point of view, families but also institutions and teachers have a great responsibility for the development of autonomy in the classroom. If this responsibility remains within strict and limited rights and freedoms for all individuals within the interest groups, one can then foresee that the educational goals to be achieved will be compromised by the institutions themselves.

\footnotetext{
${ }^{1}$ Heteronomy in this context is based on an action that is influenced by a force outside of the individual (here: families, teachers and institutions). Mostly: The opposite of autonomy. Heteronomy decreases while autonomy increases, and vice versa.
} 
For example, the obligation to use a single source in teaching will make it difficult for teachers to overcome the possible weaknesses of this textbook. It is therefore necessary to give teachers more freedom to use their own teaching materials. The variety of teaching materials and flexible teaching organization could give both teachers and learners more freedom, which could then also be helpful for autonomous learning, but which ideas still need research. From these discussions it can be concluded that learners in environments of high authority will tend to prefer passive learning. Teachers must therefore be able to balance autonomy and heteronomy in the classroom to move closer to autonomous learning.

The second part in this study presented a historical overview of the direct and indirect influences that have influenced the concept of "learner autonomy" in foreign language research. Among the most important factors influencing the development of the concept of "autonomy" are the ideals and expectations raised by the political turmoil in Europe in the late 1960s, technological developments, the growing demand for learning new foreign languages and the huge increase in the number of learners wishing to pursue higher education. These factors have contributed to grow importance of distance learning. It can be seen that parallel to population growth and economic developments, the need for education and for people who speak at least one foreign language has risen directly. While the importance of learning foreign languages as a result of these conditions has increased worldwide, the concept of autonomy has also developed at the same time. 


\section{Einleitung}

Das Interesse an der Autonomieforschung zum Sprachenlernen hat seit Mitte der 1970er Jahre zugenommen (u.a. Chan 2015: 148). Seitdem beschäftigten sich viele Fremdsprachenlehrer ${ }^{2}$ damit, dass ihre Lernenden auch außerhalb des Klassenzimmers, ihre eigene Verantwortung für ihr eigenes Lernen übernehmen.

Das Konzept der Autonomie beim Sprachenlernen hat eine Vielzahl von Ansätzen in der Theorie und Praxis des Sprachunterrichts beeinflusst und wurde auch selbst von diesen beeinflusst. Jedoch kann man hier nicht behaupten, dass sie ursprünglich ein Sprachbildungskonzept wäre (Hildt 2004: 37). Denn die „Autonomie“ bzw. „Lernerautonomie“ in der Fremdsprachendidaktik wurde des Öfteren als Forschungsgegenstand angesehen (Littlewood 1999: 71; Benson 2007: 21). Sie wurde von vielen Autoren in verschiedenen Sichtweisen interpretiert und benannt. Einige der meist auftretenden Definitionen zum Begriff „Autonomie“ sind z. B. „Verstärkung der Lernenden“, „Lernerautonomie“, „Selbstgesteuertes Lernen“ oder „Unabhängigkeit der Lernenden“. Paulo Freire, Henri Holec, Rebecca Oxford bis zu Phil Benson, hatten alle eine wirkmächtige Vorstellung und Idee über das Lernen der Lernenden, die dazu führten, dass aktives und selbstständiges Lernen an Bedeutung gewann.

Der aus der Politik und Philosophie hervorgegangene Begriff „Autonomie“ wird gewöhnlich mit „Selbstgesetzgebung“ übersetzt. Er wurde seit der Antike in verschiedenen Bereichen verwendet und ist auch noch in großem Umfang aktuell. Die Autonomie, die im altgriechischen „Autonomia“ genannt wurde, bedeutet „Eigengesetzlichkeit, Selbstständigkeit“ und setzt sich aus den Begriffen ,autós (Selbst)“ und „nomós (Recht- Gesetz)“ zusammen (Wrana 2008: 34). Dieser Begriff hatte im antiken Griechenland ursprünglich eine politische Bedeutung und wurde von Tassinari (2010: 33) in ähnlicher Weise erläutert. Tassinari (ebd.) zufolge bedeutet „Autonomie“ im altgriechischen, die Fähigkeit darüber, sich mit seinen eigenen Gesetzen regieren zu können. Herodot (ca. 490-420 v. Chr.) jedoch verwendete den Begriff „Autonomie“ etwas umfassender als ,innere und äußere politische Freiheit“. Seit seinem ersten Erscheinen im Altertum, bis in die heutige Zeit wurde das Wort „Autonomie“ vielen Bedeutungswandlungen unterworfen (Shala 2014: 5).

Der Begriff Autonomie fand in der Zeit von Aristoteles seinen Platz in der Politik, im 20. Jahrhundert dagegen erweiterte sich der Begriff und zwar sowohl im politischen als auch im philosophischen Bereich (Shala, ebd: 5). Die Wandlung des Wortes begann nach Tassinari (2010: 33) erst im 18. Jahrhundert mit der Erweiterung der rechtlichen Bedeutung von Mündigkeit der Individuen und wurde somit auf das philosophische Gebiet übertragen. Letztendlich wurde der Begriff „Autonomie“ in den verschiedenen Bereichen der Philosophie, Politik und Wirtschaft als eine innere, moralische und erkenntnistheoretische Fähigkeit des Individuums verwendet.

\footnotetext{
${ }^{2}$ Aus Gründen der einfacheren Lesbarkeit wird im Text das generische Maskulinum verwendet.
} 
Im mittelalterlichen Europa entwickelte sich die Autonomie zuerst im Bildungsbereich, in den Gilden der Meister (Lehrer) und Lehrlinge (Schüler). Später wurden diese Gilden die ersten Universitäten in Europa. Damals führten diese Gelehrtengilden ihre eigenen Geschäfte, obwohl ihre Finanzierungsquellen von Feudalherren oder dem Papsttum kamen. Im Wesentlichen waren die mittelalterlichen Universitäten selbstverwaltete und selbstfinanzierte Institutionen. Daher war die Bedeutung von Autonomie zu dieser Zeit einerseits mit der Unabhängigkeit von der Finanzierung und andererseits mit der Unabhängigkeit der Institution in der Wissenschaft verbunden (vgl. Bingöl 2012: 52f.). Im 18. Jahrhundert begann man, die Bedeutung des Begriffs um die rechtliche Bedeutung der Mündigkeit, die sich auf Individuen bezieht, zu erweitern und auf das Gebiet der Philosophie zu übertragen. Autonomie ist auch daher im Zeitalter der Aufklärung ein wichtiger Begriff. In dieser Zeit bedeutet er Freiheit und Selbstbestimmung auf politischer und individueller Ebene (Tassinari 2010: 33).

Im 20. Jahrhundert wurden Fachgebiete wie Philosophie, Psychologie, Politik und Bildung immer stärker von den Begriffen „Autonomie“ und „Unabhängigkeit“ beeinflusst (Benson und Voller 1997: 4). Laut Gremmo und Riley (1995: 151) hatte der Begriff „Autonomie“ im 20. Jahrhundert politische, soziale und philosophische Determinanten und wurde in den 1960er Jahren als Forschungsgegenstand in den Bereichen Pädagogik, Lernpsychologie und auch in der Fremdsprachenforschung angesehen. Die Bekanntheit von Begriffen wie „schülerorientiert, kommunikativer Sprachunterricht, Handlungsorientierung und handlungs- und aufgabenorientiertes Curriculum" sind beispielgebend dafür, wie sich die Lernerautonomie im Laufe der Zeit in der Pädagogik des Fremdsprachenunterrichts bemerkbar macht (vgl. Gremmo und Riley, ebd.).

Bei einer ausführlichen Literaturrecherche im Bereich des Fremdsprachenunterrichts ist festzustellen, dass sich in den letzten dreißig Jahren viele verschiedene Definitionen herausgebildet haben, die den Begriff „Lernerautonomie“ komplizierter machen und den Umriss des Begriffs nicht vereinfachen. Viele Wissenschaftler (u. a. Martinez 2005; Benson 2007; Tassinari 2010; Casas 2013) kritisieren dies, weil diese Vielfalt an Definitionen zu Missverständnissen und Unklarheiten führt. Daher versucht die vorliegende Arbeit den Begriff „Autonomie“ in den pädagogischen Strömungen durch einige Vorläufer der Autonomieidee zu erläutern. Dabei wird auch die frühe Geschichte der Autonomie in Bezug zum Fremdsprachenunterricht zur Diskussion gestellt. Auf diese Weise können Rückschlüsse darauf gezogen werden, welche Dimensionen dieses Konzepts sich im Sprachunterricht widerspiegeln sollten.

\section{Der Begriff „Autonomie“ in den pädagogischen Strömungen}

Es gibt eine Vielzahl von Definitionen für den Begriff „Autonomie“, die je nach den theoretischen Ansätzen und der jeweiligen Zeit neue Bedeutung finden. In den pädagogischen Strömungen, die als Vorläufer der Idee der Autonomie angesehen 
wurden, hatten Persönlichkeiten wie Jean-Jacque Rousseau, John Dewey, William Kilpatrick und Paulo Freire großen Einfluss auf das heutige Konzept der „Lernerautonomie“. Im Nachfolgenden werden die oben genannten Autoren bzw. Philosophen und ihre Beiträge zur Autonomie und zum Autonomiegedanken in chronologischer Reihenfolge behandelt.

\subsection{Jean-Jacque Rousseau}

Jean-Jacque Rousseaus (1712-1778), der auch als „ein Vordenker des Konstruktivismus“ bekannt ist, bezeichnete die Autonomie im einfachsten Sinne, dass man sich von keiner anderen Autorität außer dem eigenen Verstand beherrschen lassen sollte (Neuhouser 2011: 483; Tütüncü 2013: 309).

Die Konzeption der Autonomie wurde als ein persönliches und nicht rein politisches Ideal in der modernen westlichen Philosophie betrachtet und begann mit Jean-Jacques Rousseaus Werk namens „Social Contract“ (1762), in der die Bürger durch Autonomie oder Selbstgesetzgebung dazu gebracht wurden, sich zusammenzuschließen, um für sich selbst Gesetze zu errichten, die ihr kollektives Verständnis des Gemeinwohls widerspiegeln. Entsprechend dieser traditionsreichen Denkweise sind viele Aussagen darüber gemacht worden, was Autonomie ist und warum sie als wertvoll angesehen werden sollte. Infolgedessen ist das moderne Denken zu diesem Thema in verschiedener Hinsicht weit über Rousseaus Konzept der Autonomie hinausgegangen. Im Laufe der Zeit hat sich das Autonomiekonzept zu einem Ideal der Selbstkontrolle entwickelt. Rousseau zufolge erfordert die Autonomie Individuen, die $\mathrm{zu}$ einem unabhängigen moralischen Urteil fähig sind. Einfacher formuliert bedeutet dies, dass die Autonomie von den Individuen erforderte, die Fähigkeit für sich selbst zu urteilen, auch die Bereitschaft, eine misstrauische Haltung gegenüber ihren eigenen moralischen Urteilen einzunehmen, besonders dann, wenn sie sich von anderen unterscheiden wollten. Man kann diese Haltung gegenüber sich selbst auch als eine Haltung beschreiben, die man gegenüber anderen einnimmt. Autonomie zu erlangen erfordert eine Haltung des Respekts vor den Urteilen anderer (Neuhouser 2011: 490-491).

Ein Werk von Rousseau, welches „Emile“ oder auch „Über die Erziehung“ (1762) genannt wird, ist auch bedeutsam für die Autonomie und ihre Diskussion. Die Idee zur Bildung wird überwiegend in dem Werk von Rousseau „Emile“ dargelegt, in dem die Erziehung so weit wie möglich in Harmonie mit der Entwicklung der natürlichen Fähigkeiten des Kindes durch einen Prozess der autonomen Entdeckung erfolgen sollte. Dafür musste Emile an der Gesellschaft und dem sozialen Milieu teilhaben und darin leben. Aber Emile musste vorsichtig sein, denn Emile hätte von anderen Mitmenschen beeindruckt werden können, oder in die Falle seiner Leidenschaft und seines Ehrgeizes geraten können (Rousseau 2011: 351). Um solche Risiken, die von dem sozialen Milieu hervortreten können, entgegenzukommen, hielt man es für wichtig, als autonome Person aufzuwachsen. Aus diesem Grund musste Emile wissen, dass das einzige Licht, das ihn leitet, sein eigener Verstand war (Kabasakal 2017: 115) und 
nichts anderes. Wie am Beispiel von Emiles Erziehung zu sehen ist, betont Rousseau den direkten Kontakt mit der Natur ${ }^{3}$, wenn es um das Lernen geht. Wenn man diese Konstellation auf den Fremdsprachenunterricht überträgt, würde daraus die Notwendigkeit für die Sprachenlernenden deutlich, die Zielsprache in einer authentischen Umgebung zu lernen.

Laut Casas (2013: 18) führte dieses Werk „Emile“ zu einer pädagogischen Verwirrung: Während Emile in diesem Bildungsroman das Recht dazu hatte, unabhängig zu leben und zu handeln, so müsste dieser Bildungsweg nach Rousseau in hohem Maße überprüft werden. Nach dem Werk wird diese unkontrollierte Freiheit als ein gewisses Unbehagen empfunden. Die Freiheit sollte laut Rousseau im Bildungsprozess eingeschränkt und kontrolliert werden. Anders formuliert sollte nach ihm Emile ein guter Bürger sein und dazu erzogen werden. In dieser Hinsicht versteht Jean-Jacque Rousseau unter dem Begriff „Erziehung“ eine Synthese aus autonomem Handeln und der Verbundenheit mit der Nation.

\subsection{John Dewey}

John Dewey gilt als der „Vater“ der Projektmethode. In der angloamerikanischen Diskussion wird Dewey (1859-1952) heute von vielen Pädagogen als der reformistische Klassiker des 20. Jahrhunderts angesehen. Aus pädagogischer Sicht war es John Dewey, der in seiner Publikation „Democracy and Education“ den Grundstein für die Entwicklung des Konzepts der Lernautonomie legte (Neubert 2004: 21).

Deweys Bildungsphilosophie geht davon aus, dass jeder einzelne Mensch eine moralische Verantwortung dazu tragen soll, sich an der Entwicklung und Reform der Gesellschaften, in denen sie leben, zu beteiligen. In dieser Bildungsphilosophie wird angenommen, dass Entwicklung selbst das Ziel von Bildung ist und, dass die Transformation des Selbst eine bedeutende Rolle spielt (Dall'Acqua 2018: 8). Dewey war der Ansicht, dass der Schulbesuch auf diese Art und Weise nicht allein auf die Zukunft vorbereiten sollte, mit denen die Schüler später im Leben konfrontiert werden. Stattdessen sollte es vielmehr darum gehen, aktuelle Probleme zu lösen, weshalb er argumentierte, dass Bildungsaktivitäten von der unmittelbaren persönlichen und sozialen Lernerfahrung ausgehen sollten. Nach Deweys Ansicht ist der Lernprozess ein adaptiver Prozess, bei dem die Interaktion mit der Außenwelt Probleme schafft, die gelöst werden müssen, damit der Einzelne seine Bedürfnisse erfüllen kann. Diese Sicht des Lernens als adaptiver Prozess ist auch die Wurzel der konstruktivistischen Lernansätze, die einen entscheidenden Anstoß für die Autonomietheorie des Sprachenlernens gegeben haben (vgl. Benson 2011: 29).

Die Beziehung zwischen Schule und Gesellschaft sollte Dewey (1896) zufolge, in Theorie und Praxis vor dem Hintergrund nachhaltiger sozialer Veränderungen durch

\footnotetext{
${ }^{3}$ Der Naturzustand ist der Ausgangspunkt für die meisten Theorien zum Gesellschaftsvertrag und ist eine abstrakte Idee. Rousseau stellt sich einen Menschen vor, der im Naturzustand den Einklang mit der Natur suchte (siehe Massimo 2017).
} 
Industrialisierung und städtischen Wandel neu bestimmt werden. Von besonderer Bedeutung sei es, die Schule auf das Leben des Kindes zu fokussieren, um unnötige Energieverschwendung zu vermeiden. Dewey erwähnt, im Zusammenhang mit den notwendigen Veränderungen in Schule und Unterricht, auch eine Veränderung für die Pädagogik, die eine mit der kopernikanischen Revolution in der Astronomie vergleichbare Revolution repräsentiert. Das Kind wird in diesem Fall zur Sonne, um die sich die Erziehungshilfen drehen; es ist im Mittelpunkt, um den sie herum organisiert sind (zit. n. Neubert 2004: 21). Neubert (ebd.) betont außerdem, dass Lernen für Dewey immer in der Mitte des Lebens beginnt und durch ,aktives Experimentieren, Konstruieren, Ausprobieren, Beobachten und Diskutieren in Zusammenarbeit mit anderen Lernenden" stattfindet.

\section{(...), womit ein Lernen durch aktives Experimentieren, Konstruieren, Ausprobieren, Beobachten und Diskutieren in Kooperation mit anderen Lernenden besonders betont wird. (Neubert 2004: 21)}

Dewey weist auch auf die Notwendigkeit hin, eine gemeinschaftliche Unterrichtsumgebung zu schaffen, die die kontinuierliche Entwicklung des kindlichen Lernens fördert und nicht den bloßen Erwerb von Wissen und Fächern. Somit setzte er sich methodisch dafür ein, die Studie aus der Praxis heraus zu realisieren. Die idealen Werte sollten nicht auf die Erfahrung übertragen werden, sondern aus der Erfahrung selbst entstehen und wieder zurückkehren, um nachfolgende Erfahrungen zu leiten (Benson 2001: 28; Wilson und Ryg 2015: 132). Laut Dewey sollte der Hauptzweck der Bildung in einer demokratischen Gesellschaft darin bestehen, die Lernenden auf die aktive Teilnahme am sozialen und politischen Leben vorzubereiten, indem ihnen geholfen wird, die Fähigkeiten und Einstellungen zu erwerben, die sie brauchen, um demokratisch und sozial zu sein (Benson 2001: 29). Die Institution ,Schule“ spielt eine bedeutsame Rolle im Zentrum seines pädagogischen Denkens, da die Lernenden dort kritisches Denken und die Grundlagen der Demokratie erlernen. Casas (2013) erklärt in seiner Arbeit die Bedeutung der Erziehung für die demokratische Entwicklung und Deweys pädagogisches Denken, indem er auf die Teilnahme an sozialen und politischen Ereignissen hinweist:

\footnotetext{
Persönliche Erfahrung und soziale Interaktion würden die lebensweltlichen Voraussetzungen und das Mittel eines über den ,Stoff' hinausgehenden Lernens darstellen. Teilhabe am sozialen und politischen Geschehen sowie der Anspruch, an Veränderungsprozessen und an Lösungen sozialer Missstände mitzuwirken, charakterisieren den von Dewey entworfenen, gebildeten, mündigen Bürger. (Casas 2013: 20$)^{4}$
}

Nach der detaillierten Darstellung von Deweys Konzept wird die Sicht von Wilson und Ryg (2015) auf Deweys Autonomiekonzept aufbauend diskutiert, so dass diese Art der Betrachtung verschiedener Darstellungen aus unterschiedlichen Perspektiven ein ganzheitlicheres Verständnis ermöglicht. Wilson und Ryg (2015: 137) zufolge war Autonomie für Dewey kein Ideal, vielmehr ein kontinuierlicher Prozess der Aushandlung einer kritischen und einsichtsreichen Auseinandersetzung mit den

\footnotetext{
${ }^{4}$ Diese und nachfolgende Zitate wurden von Y.E.S. ins Deutsche übersetzt.
} 
Lebensbedingungen. Für Dewey waren Ideale keine äußeren Ziele; sie ergaben sich aus der Untersuchung bestehender Gesellschaften und menschlicher Praktiken. Begrifflichkeiten wie „Selbst“, „Individuum“ und „Person“, die sich leichter integrieren und in der Erfahrung einordnen ließen, zog Dewey anstatt abstrakten Begriffen wie „Autonomie“ vor. Außerdem verwendete Dewey den Begriff, um die Deontologie Immanuel Kants zu erklären. Die Autonomie wurde in all diesen Verfahren als deskriptives Adjektiv und nicht als eigenständiger Forschungsbegriff verwendet (Wilson und Ryg 2015: 142).

\subsection{William Kilpatrick}

Als ein wichtiger Anhänger der nordamerikanischen progressiven Erziehungsbewegung hat der Name William Kilpatrick (1871-1965) einen besonderen Wert. Kilpatrick vertrat die Anschauungen von Rousseau (Benson 2001: 30). Er setzte sich dafür ein, dass sich seine Methoden für Gruppenarbeiten im Lehr- und Lernverfahren eignen. Durch diese Gruppenarbeiten sollten Lernende Fähigkeiten und Einstellungen erwerben, die für eine demokratische, soziale Teilhabe erforderlich sind (Benson 2001: 31). Diese Herangehensweise sollte zur Demokratie der höheren Bildung beitragen. Es lag in ihrem Interesse die Form des Studiums zu ändern und anstatt des rezeptiven Lernens ein immer mehr analytisches, experimentvolles und kreatives Lernverfahren durchzuführen (Knoll 1998: 350).

Kilpatrick leistete einen großen Beitrag zur Idee der Autonomie durch sein Werk „Projektmethode“, die im frühen 18. Jahrhundert begann. Die Studenten der Académie Royale d'Architecture in Paris mussten nachweisen, dass sie für die Anwendung der Prinzipien von Gestaltung und Konstruktion geeignet waren. In der „Projektmethode“ planen und führen Studenten ihre eigenen Lernprojekte aus. Daher wurden sie in regelmäßigen Abständen aufgefordert, Pläne für ein „Projekt“", d. h. ein Bauprojekt, wie zum Beispiel ein Denkmal, eine Kirche, eine Reithalle, einen Palast, auszuarbeiten (Knoll 2012: 4) und durchzuführen.

Die Bewegung, die durch W. Kilpatrick und J. Dewey entstand, hat die Möglichkeit geschaffen, die Lernerautonomie beim Fremdsprachenerwerb zu fördern. Die theoretische Diskussion über Lernerautonomie war allerdings damals weit davon entfernt, einheitlich, verständlich und systematisch zu sein (Teng 2019: 2).

\subsection{Paulo Freire}

Paulo Freire (1921-1997), der im Jahre 1970 sein Werk „Die Pädagogik der Unterdrückten“ veröffentlichte, war ein brasilianischer Pädagoge und Philosoph. Dieses Werk wird als einer der jüngeren Klassiker der Pädagogik angesehen und gehört zu den zentralen Werken der kritischen Erziehungswissenschaft. Das in der spanischen Sprache veröffentlichte Werk wurde später auch ins Englische, Italienische, Französische und Deutsche übersetzt. 
Die Theorien von Freire führten auch in den europäischen Ländern, wie z. B. in Deutschland, zu Diskussionen in pädagogischen und psychosozialen Disziplinen (Herriger 2006: 34). Freire (1970) betont in seinem Werk „Die Pädagogik der Unterdrückten“ den Unterschied zwischen Bank- und Problemausbildung und benennt die Unterrichtsmethode in diesen Jahren als „Bankiers-Methode“. Freire kritisiert dadurch den Positivismus und die Anhänger dieses Gedankens seiner Zeit, die das menschliche Bewusstsein als passiv und leer qualifizieren. Darüber hinaus versucht Freire diese positivistische Philosophie mit seiner eigenen Methode darzulegen. Die Erziehung selbst wird in seiner Bankiers-Methode zu einem Akt der Spareinlage. In dieser genannten Bankiers-Methode macht der Lehrer, wie in einer Sparbüchse, Einzahlungen in die Köpfe der Schüler, mit dem Ziel, dass die Köpfe der Schüler ,,mit den Inhalten seiner Übermittlung“ gefüllt werden. Allerdings tritt in diesem Konzept nach Freire ein Problem auf. Die Inhalte, die von den Schülern gelernt werden sollen, sind von der Wirklichkeit entfernt. Sie haben somit keine Verbindung zu ihrer Lebenserfahrung. Aus diesem Grund macht die Bankiers-Methode die Schüler passiv, trägt nicht zur Entwicklung des kritischen Bewusstseins bei und führt sogar nach Freire dazu, durch diese Methode unterdrückt zu werden. Laut Hahn (2012: 62) kommt diese Erniedrigung der Schüler dadurch zustande, dass sie des Öfteren hören, dass aus ihnen nichts wird und unfähig sind, etwas zu lernen, wonach sie dann selbst zu ihrer eigenen Unfähigkeit überzeugt werden.

In diesem Zusammenhang betonen Aliakbari und Faraji (2011: 78) das Freire (1970) sich metaphorisch auf die traditionelle Anschauung von Bildung als Bankmodell bezieht, weil es sich dem der Anzahlung in einer Bank ähnelt. Nach ihnen spiegelt dieses Modell die Struktur einer unterdrückenden Gesellschaft wider, in der es sowohl Unterdrückte als auch Unterdrückende gibt. Darum betonen Aliakbari und Faraji (2011), dass dieses Modell abgelehnt werden sollte. Der Grund dafür liegt nach ihnen darin, in dem die Lehrer doch beauftragt sind, um für die Gesellschaft zu sorgen den Menschen, bzw. den Schülern Gelegenheit dazu zu geben, die Position innerhalb der Gesellschaft kritisch zu reflektieren und in ihr zu handeln.

Daraus lässt sich ableiten, dass in der traditionellen Auffassung der Bildung Lehrer diejenigen sind, die das Wissen formen und den Schülern übertragen und nicht in der Erwartung sind, dass die Schüler das übertragene Wissen in Frage stellen.

Nach Miethe (2016) tritt in Freires Auffassung ein erweitertes Bildungsverständnis vor, das sich nicht nur auf die individuelle Erziehung bezieht, sondern auch auf die Gesellschaft insgesamt. Die Grundthese von Freire wird von Miethe mit dem Satz: „Erziehung ist niemals neutral“, kurz zusammengefasst.

Die Vertreter der kritischen Theorie sind der Auffassung, dass die Ziele nur erreicht werden können, wenn die unterdrückten Menschen emanzipiert werden, was sie dann dazu bringen kann, ihre Lebensbedingungen zu verändern. Freire widmete sich an bestimmte Gruppen der Gesellschaft, wie die der Behinderten, Alten, Kinder und Analphabeten (Casas 2013: 22). Um die Bauern in den Prozess der Schulung (Alphabetisierung) einzubeziehen, empfahl Paulo Freire (1970), Themen und Lektionen 
zu konstruieren, die auf Diskussionen mit den Bauern eingehen und auf der Grundlage ihrer Welt und ihrer Probleme beruhen.

Paulo Freires Interesse (Funke 2010: 18-19) galt der Lernerautonomie mit einem moralischen Aspekt, welches er mit einem politischen Konzept zu verbinden versuchte. Er vertrat die Anschauung, dass autoritäre politische Systeme auf dem entpolitisierenden Einfluss der Massenbildung beruhten und durch radikale Bildungsreformen in Frage gestellt werden könnten. Sein Hauptanliegen lag darin, dass durch Bildung ein kritisches Bewusstsein gefördert werden kann, welches dem Menschen ermöglicht, ihre eigenen Lebensumstände in Frage $\mathrm{zu}$ stellen und sie womöglich auch zu ändern.

Laut Freire war Lernerautonomie vor allem die individuelle Einzigartigkeit jedes Einzelnen (2008: 55), in dem die sozialen, politischen und kulturellen Zusammenhänge zum Lernen eingebettet waren (vgl. auch Freire 2008: 97). Dabei war Bildung nach seiner Ansicht niemals ein neutraler Prozess, da sie unter dem Einfluss des Politischen lag. Dieser politische Einfluss kann nach ihm niemals von der Pädagogik getrennt betrachtet werden. Auf diese Feststellung aufbauend vertritt Freire die Meinung, dass es für Mitglieder einer Gesellschaft äußerst wichtig ist, sich der eigenen Handlungen bewusst zu werden (vgl. Diaz 2011: 2) und danach zu handeln.

Der Begriff „Autonomie“ wurde in den pädagogischen Strömungen unter Berücksichtigung einiger Stellvertreter wie J. J. Rousseau, J. Dewey, W. Kilpatrick und P. Freire näher betrachtet, anschließend wird die Entwicklung des Konzepts der Autonomie auf dem Gebiet der Fremdsprache erörtert.

\section{Der Begriff „Autonomie“ und sein geschichtlicher Hintergrund im Fremdsprachenunterricht}

Dieses Kapitel widmet sich dem Autonomiegedanken und ihren Auswirkungen auf den Sprachunterricht, in dem versucht wird, die frühe Geschichte der Autonomie in Bezug zum Fremdsprachenunterricht darzulegen.

In den vergangenen Jahren ist die Anzahl der Publikationen zum Thema „Autonomie, autonomes Lernen und Lernerautonomie“ im Bereich der Fremdsprachen stetig gestiegen. Ende der 1970er Jahre entstanden die ersten entscheidenden Ansätze für die Debatte über die Lernerautonomie in der Fremdsprachenforschung (Benson 2007: 21; Little 2007: 1; Teng 2019: 1). Aber mehr denn je findet der Begriff „Autonomie“ seinen Platz in der Bildungsdiskussion. Auch die Publikationen zu diesem Thema stoßen auf ein zunehmendes Interesse und gewinnen an Wert. Da der Begriff „Autonomie“ in komplexen Zusammenhängen mit Entwicklungen in der Philosophie, der Politikwissenschaft, der Psychologie und der Soziologie steht, die zum Teil viele Jahrhunderte zurückreichen, kann der Begriff „Autonomie“ keinesfalls auf eine einzige Informationsquelle oder ein bestimmtes Ursprungsdatum zurückgeführt werden. Da sowohl „Sprache“ als auch „Lernen“ ein breites Spektrum an Phänomenen beeinflussen 
und zugleich auch von vielen beeinflusst werden (Little 1991: 1f.; Gremmo und Riley 1995: 152).

Um einen Überblick über die Faktoren oder Beziehungen zwischen den verschiedenen Definitionen von Lernerautonomie in der Fremdsprachenforschung zu erhalten, werden die direkten und indirekten Einflüsse mit besonderem Interesse durch die Arbeit von Gremmo und Riley (1995) im Folgenden dargestellt, da sie dieses Thema im Gegensatz zu anderen Wissenschaftlern unter verschiedenen Aspekten ausführlich behandelt haben.

Laut Gremmo und Riley (1995: 152) wurden die Vorstellungen von „Autonomie“ und „Selbststeuerung“ in den 20 bis 25 Jahren nach dem Zweiten Weltkrieg zum Gegenstand einer intensiven Prüfung, Analyse und Debatte. Das Interesse am Autonomiekonzept im Sprachunterricht war zum Teil eine Antwort auf die Ideale und Erwartungen der Menschen für ein besseres und demokratisches Leben, die durch die politischen Auseinandersetzungen in Europa in den späten 1960er Jahren geweckt wurden.

Nach Auffassung der Autoren (ebd.) lassen sich einige wichtige Aspekte und Ereignisse, die dazu führten, dass der Begriff „Autonomie“ und seine Anwendbarkeit im Sprachunterricht zunehmend an Bedeutung gewann, in sechs Überbegriffen festhalten:

Der erste Faktor lautet „Die Welle der Bewegung für Minderheitenrechte“. Hier geht es darum, die Werte, Motive und Ziele von „Emanzen“, „Konsumenten“, „Ökologen“ und „Angehörigen vieler ethnischer, religiöser und sprachlicher Minderheiten“ zu schützen und sicherzustellen, dass diese Gruppen ihre Rechte besitzen und ein autonomes Leben erhalten (Gremmo und Riley 1995: 152).

Aufbauend darauf diskutiert der zweite Faktor „Die Reaktion gegen den Behaviorismus", in der die westliche Gesellschaft den Behaviorismus in einigen Punkten kritisiert und als Folge dieser antideterministischen Haltung die Suche nach „Alternativen“ aller Art begann: alternative Lebensstile, Medizin, Politik, Musik, Poesie und Erziehung.

Der von den Autoren als „das Interesse an Minderheitenrechten“ bezeichnete dritte wichtige Faktor hatte einen direkten Einfluss auf die Entwicklung der Erwachsenenbildung in Europa. Die wohl wichtigste Ausprägung dieses Einflusses war das 1971 gegründete Projekt „Moderne Sprachen“ des Europarates, das sich auf die Sprachbedürfnisse der Migranten konzentrierte. „Autonomie“ war von Anfang an ein wichtiges Element im Gesamtrahmen der Arbeit des Europarates. Der Grund dafür war zum Teil die Folge des kommunikativen Ansatzes, zum Teil die Anwesenheit von Yves Chalon im Sachverständigenausschuss. Chalon als Gründer des CRAPEL ${ }^{5}$ initiierte eine

\footnotetext{
${ }^{5}$ CRAPEL: Centre de Recherches et d'Applications en Langues (Dt. Übersetzung: Zentrum für Forschung und Anwendungen in Sprachen)
} 
Reihe von Projekten, die „Autonomie“ und „Selbstkontrolle“ umsetzten und unter Henri Holec weitergeführt wurden (Gremmo und Riley 1995: 153).

Die Autoren wiesen als den vierten Faktor auf die technologischen Entwicklungen hin, die unbestreitbar zur Verbreitung von „Autonomie und Selbsterfolg“ beigetragen hatten. Dieser Faktor betont die Zunahme des Verbraucherbewusstseins, die sich auf die Wahrnehmung der Rolle des Lernenden auswirkte. Dabei wurde der Lernende nicht mehr als passiver Empfänger institutioneller Wohltätigkeit gesehen, sondern als ein Verbraucher, der auf dem Markt sachkundige Entscheidungen trifft.

Als fünfter Faktor ist die Nachfrage nach Fremdsprachen zu nennen, die sowohl durch politische Entwicklungen (Europäische Union, Vereinte Nationen) und den Aufstieg multinationaler Konzerne (wie Unilever, IBM, Renault, Shell) als auch einfache Mobilität und Tourismus stark gestiegen ist.

Der letzte Faktor befasst sich mit dem enormen Wachstum der Bevölkerung in Schulen und Universitäten. Durch den breiteren Zugang zur Bildung, der mit der hohen Anzahl von Lernenden in Schulen und Universitäten zusammenging, haben sich in vielen Ländern neue Bildungsstrukturen wie Fernstudium oder Fernschule herausgebildet, um mit einer so großen Anzahl von Lernenden umgehen zu können. In diesem Zusammenhang wurde das selbstgesteuerte Lernen in zahlreichen Institutionen mit institutioneller Unterstützung in Form von Beratungs- und Ressourcenzentren als nützlich angesehen (Gremmo und Riley 1995: 154).

\section{Schlussfolgerung}

Aufgrund der Tatsache, dass der Begriff „Autonomie“ im Ursprung unterschiedliche Bedeutungen hat und in verschiedenen Bereichen (wie Psychologie, Philosophie, Politik usw.) verwendet wird, hat er im Bereich der Fremdsprache zu einigen Bedeutungsunterschieden geführt.

Um die Bedeutung von „Autonomie“ beim Fremdsprachenlernen besser zu verstehen, wurde es als wichtig angesehen, wie der Begriff „Autonomie“ entstanden ist und warum ihm heutzutage große Bedeutung gewidmet wird. Dieser Artikel beschäftigt sich aus diesem Grund mit der historischen Entwicklung und der Bedeutung des Wortes „Autonomie“.

In der vorliegenden Studie wurde der Begriff „Autonomie“ in den pädagogischen Strömungen durch einige berühmte Vorläufer und ihre Konzeptionen der Autonomieidee wie „Jean-Jacque Rousseau“, „John Dewey“, „William Kilpatrick“ und „Paulo Freire“ zur Sprache gebracht, da sie großen Einfluss auf das heutige Konzept der „Lernerautonomie“ hatten. Diese erwähnten Autoren prägten mit ihren Konzeptionen und Werken die Konstruktivismus-Theorien und die auf ihrer Grundlage konzipierten Methoden. 
Bei den konstruktivistischen Ansätzen wird die Annahme vertreten, dass Lernen ein konstruktiver Prozess ist und es wird behauptet, dass jeder Lernende aus seinen eigenen Erfahrungen lernt, indem er seine eigenen Werte, Überzeugungen und früheren Erfahrungen nutzt. Man kann dann davon ausgehen, dass all diese persönlichen Erfahrungen und Kenntnisse die Lernenden sowohl in Bezug auf ihre moralische als auch auf ihre Lernfähigkeiten gereift haben (Textor 2000; Kron und Reddig 2003).

Das Lernen im Sprachunterricht im Kontext der konstruktivistischen Ansätze basiert auf langjährigen Erfahrungen in und außerhalb des Klassenzimmers und der Wissensbearbeitung im Lernprozess (Güneş 2010: 5; İlhan, Gülersoy, Çelik 2017: 61). Wie im heutigen modernen Verständnis des Fremdsprachenlernens bedeutet dies, dass die Lernenden durch Erfahrung und Erleben lernen, und nicht durch Auswendiglernen. Gesucht sind zudem Lehrmethoden, die die Diversität der Lernenden in Bezug auf intellektuelle Fähigkeiten und Lernpotenzial besser berücksichtigen. $\mathrm{Zu}$ diesen Lehrmethoden gehören z. B. Binnendifferenzierung, Handlungsorientierter Unterricht, Problemorientiertes Lernen, Offener Unterricht, Stationenarbeit, Projektarbeit, Forschend-entwickelnder Unterricht. Aktuelle wissenschaftliche Studien haben zudem gezeigt, dass solch eine Betrachtungsweise an das Lehren und Lernen einer Fremdsprache positive und bessere Ergebnisse leisten und sowohl die kognitiven als auch die affektiven Eigenschaften der Lernenden verbesserten (Kılıç 2004: 309; Raya 2014: 155).

Solche Methoden können aber nicht in einem Bildungsmodell stattfinden, in dem die Lehrenden eine autoritäre Figur darstellen, in dem Wissen und Fertigkeiten den Lernenden nach einem vorgegebenen Lehrplan vermittelt werden. Auf dieser Grundlage ist klar, dass der Mangel an Autonomie in der Lerngruppe umso größer sein wird, je mehr Heteronomie in den Klassenraum gelangt. Unter diesem Gesichtspunkt tragen die Institutionen und Lehrenden für die Entwicklung der Autonomie im Klassenzimmer eine große Verantwortung. Wenn diese Verantwortung innerhalb strikter und begrenzter Rechte und Freiheiten für alle Individuen innerhalb der Interessengruppen bleibt, kann man dann voraussehen, dass die zu erreichenden Bildungsziele durch die Institutionen selbst beeinträchtigt werden. Zum Beispiel wird die Verpflichtung, sich nur an eine einzige Quelle beim Unterrichten zu halten, den Lehrenden Schwierigkeiten bereiten, die möglichen Schwächen eines einzigen Lehrbuchs zu überwinden. Es ist daher notwendig, den Lehrenden mehr Freiraum für die Einsetzung ihrer eigenen Unterrichtsmaterialien zu geben. Die Vervielfältigung der Unterrichtsmaterialien und flexible Unterrichtsorganisation könnten sowohl Lehrenden als auch Lernenden mehr Freiraum leisten, was dann auch das autonome Lernen fördern könnte. Diese Gedanken bedürfen aber einer intensiveren Forschung. Aus diesen Diskussionen lässt sich schließen, dass Lernende in Umgebungen mit hoher Autorität dazu neigen werden, passives Lernen zu bevorzugen (vgl. Little 1991; Textor 2000; Yazic1 2016: 4). Die Lehrkräfte müssen daher ein Gleichgewicht zwischen der Autonomie und Heteronomie im Klassenzimmer herstellen können, um dem autonome Lernen näher zu kommen.

Der zweite Teil in dieser Studie gab einen historischen Überblick über die direkten und indirekten Einflüsse, die das Konzept „Lernerautonomie“ in der 
Fremdsprachenforschung beeinflusst haben. $\mathrm{Zu}$ den wichtigsten Faktoren, die die Entwicklung des Begriffs „Autonomie“ beeinflussen, gehören die Ideale und Erwartungen, die durch die politischen Turbulenzen in Europa in den späten 1960er Jahren geweckt wurden, die technologischen Entwicklungen, die steigende Nachfrage nach neuen Fremdsprachen und die starke Zunahme der Anzahl der Lernenden, die eine Hochschulausbildung absolvieren wollen. Diese Faktoren haben zu einer wachsenden Bedeutung des Fernstudiums beigetragen. Hier lässt sich feststellen, dass parallel zu dem Bevölkerungswachstum und den wirtschaftlichen Entwicklungen der Bedarf an Bildung und an Menschen, die mindestens eine Fremdsprache beherrschen, direkt gestiegen ist. Während diese Umstände die Bedeutung des Erlernens einer Fremdsprache in der ganzen Welt erhöhten, ermöglichten sie auch zugleich die Entwicklung des Autonomiekonzepts. So hat sich zum Beispiel Moore (1973) mit der Theorie von „Independent Learning“ sowohl mit dem Konzept der Autonomie als auch mit dem des Selbstlernens auseinandergesetzt (Horzum 2011: 1572). Laut Jones (2001) führt ein solcher Ansatz, der mehr Kontrolle über Lernprozesse ermöglicht, zu einer geringeren Abhängigkeit von Lehrern, was wiederum eine höhere Lernerautonomie zur Folge hat.

Man kann aus dieser Literaturrecherche den Schluss fassen, dass die größte Auswirkung auf die Entwicklung von Lernerautonomie in den letzten Jahren die rasante Entwicklung der Technologie ist. Mit diesem großen Einfluss der Technologie erreichte man viele Vorteile, an die man vor der Zeit des Internets nie gedacht hätte. Hierzu ist es besonders zu betonen, dass durch die neue Technologie die Bildung nicht unbedingt immer am selben Ort und zur selben Zeit stattfinden muss. Die Entwicklung sowohl der Theorie als auch der Praxis der Autonomie in den letzten Jahren durch Web 2.0 und Web-Ressourcen, hat zur Entstehung eines neuen pädagogischen Berufsstandes geführt und zwar dem des „Beraters“ (Helfer, Tutor, usw.) (Jones 2001; Hurd 2005; Benson 2011). So bedingt der Begriff „Autonomie“ heutzutage einer neueren Definition, in der beide Seiten die der Lerner sowie die Seite der Lehrer mit der Technologie und ihren Entwicklungen mit inbegriffen werden. Hierbei kann der Nutzen von empirischen Studien einen gültigen und zuverlässigen Beitrag zur Wissenschaft leisten.

\section{Literaturverzeichnis}

Aliakbari, Mohammed/ Faraji, Elham (2011): Basic principles of critical pedagogy. In: 2nd International Conference on Humanities, Historical and Social Sciences IPEDR Vol. 1, 78-85.

Benson, Phil/ Voller, Peter (1997): Autonomy and independence in language learning. London: Longman.

Benson, Phil (2001): Teaching and Researching Autonomy in Language Learning. Harlow: Pearson Education.

Benson, Phil (2007): Autonomy in language teaching and learning. In: Language Teaching, 40(1), 21-40.

Benson, Phil (2011): Teaching and Researching: Autonomy in Language Learning (Applied Linguistics in Action). Great Britain: Taylor and Francis. Kindle Edition. 
Bingöl, Bilge (2012): Üniversite özerkliğinin değişen tanımı ve üniversitelerin yeniden yapılandırılması. In: Hacettepe Hukuk Fakültesi Dergisi 2 (2), 39-75.

Casas, Alfonso Mejia (2013): Wenn und wie Lernerrollen ausgehandelt werden. Unveröffentlichte Dissertation. Universität Bielefeld: Fakultät für Linguistik und Literaturwissenschaft Lehr- und Forschungsgebiet Deutsch als Fremdsprache.

Chan, Mable (2015): Language Learner Autonomy and Learning Contract: A Case Study of Language Majors of a University in Hong Kong. In: Open Journal of Modern Linguistics 5 (2015), 147-180.

Dall'Acqua, Alessandro (2018): Die Bildung des moralischen Selbst: zur Rekonstruktion der Pädagogik des Werdens bei John Dewey. Unveröffentlichte Dissertation: University of Zurich.

Diaz, Kim (2011): Paulo Freire and his Pedagogy of the Oppressed. https://www.academia.edu/27249573/Paulo_Freire_and_his_Pedagogy_of_the_Opressed (letzter Zugriff: 24.03.2019).

Freire, Paulo (1970): Pedagogy of the oppressed. NY, USA: Bloomsbury publishing.

Freire, Paulo (2008): Pädagogik der Autonomie. Notwendiges Wissen für die Bildungspraxis. Münster: Waxmann.

Funke, Kira (2010): Paulo Freire, Werk. Wirkung und Aktualität. Münster: Waxmann Verlag.

Güneş, Firdevs (2010): Eğitimde yapılandırmacı yaklaşımla gelen yenilikler. In: Ĕ̆itime Bakış Dergisi, Ocak-Şubat-Mart, 16, 3-10.

Gremmo, Marie Jose/ Riley Philip (1995): Autonomy, self-direction and self-access in language teaching and learning. In: The history of an idea 23.2 (1995), 151-164.

Hahn, Marco: Paulo Freire (2012): Bewusstwerdung ermöglichen. Rosa-Luxemburg-Stiftung Manuskripte 97, 61. Zum Verhältnis von (politischer) Bildung und Befreiung. Manuskripte, 97.

Herriger, Norbert (2006): Empowerment in der sozialen Arbeit. Eine Einführung. Stuttgart: Kohlhammer.

Hildt, Elisabeth (2004): Autonomie. Von den Implikationen eines politisch-moralischen Ideals in Humangenetik und Neurowissenschaften. In: Jahrbuch für christliche Sozialwissenschaften 45, $37-60$.

Horzum, Mehmet Barış (2011): Transaksiyonel Uzaklık Algısı Ölçeğinin Geliştirilmesi ve Karma Öğrenme Öğrencilerinin Transaksiyonel Uzaklık Algılarının Çeşitli Değişkenler Açısından İncelenmesi, Kuram ve Uygulamada Eğitim Bilimleri, 1571-1587, http://toad.edam.com.tr/sites/default/files/pdf/transaksiyonel-uzaklik-algisi-olcegi-toad.pdf, erişim tarihi: (Letzter Zugriff: 01.07.2019).

Hurd, Stella (2005): Autonomy and the distance language learner. In: Holmberg, Boerje; Shelley, Monica and White, Cynthia eds. Distance education and languages: evolution and change. New perspectives on language and education. Clevedon, UK: Multilingual Matters, 1-19.

İlhan, Ali/ Gülersoy Ali Ekber/ Çelik Mehmet Ali (2017): Yapılandırmacı yaklaşım çerçevesinde coğrafya öğretiminde sorgulama temelli öğrenme. In: Akademik Sosyal Araştırmalar Dergisi, Yı1: 5, Say1: 43, Nisan 2017, 59-78.

Jones, Jeremy (2001): CALL and the teacher's role in promoting learner autonomy. In: CALL-EJ Online, 3(1),1. http://callej.org/journal/3-1/jones.html. (letzter Zugriff: 12.11.2020).

Kabasakal Badamchi, Devrim (2017): Jean-Jacques Rousseau'nun Eğitim Anlayışının Temelleri: Birey Emile mi, Vatandaş Emile mi?. In: Beytulhikme An International Journal of Philosophy 7 (1), 107-127. 
Kılıç, Ebru (2004): Durumlu öğrenme kuramının eğitimdeki yeri ve önemi. In: Gazi Eğitim Fakültesi Dergisi, 24 (3), 307-320. http://www.gefad.gazi.edu.tr/download/article-file/77307 (letzter Zugriff: 12.11.2020).

Knoll, Michael (1998): Zwischen bürgerlicher Demokratie und demokratischem Kollektivismus. Die amerikanische 'progressive education' in ihren politischen Optionen. In: Rülcker T. und Oelkers J. (Hg.): Politische Reformpädagogik, 349-378. Frankfurt: Lang.

Knoll, Michael (2012): I had made a mistake: William H. Kilpatrick and the project method. In: Teachers College Record 114(2) (2012), 1-45.

Kron, Thomas/ Reddig, Melanie (2003): Der Zwang zur Moral und die Dimensionen moralischer Autonomie bei Durkheim. In: Macht und Moral. VS Verlag für Sozialwissenschaften, 165-191.

Little, David (1991): Learner Autonomy 1: Definitions, Issues and Problems. Dublin: Authentik.

Little, David (2007): Language learner autonomy: Some fundamental considerations revisited. In: Innovation in Language Learning \& Teaching, 1(1), 14-29.

Littlewood, Wiliam (1999): Defining and developing autonomy in East Asian contexts. In: Applied Linguistics 20(1) (1999), 71-94.

Mori, Massimo (2017): Vom Naturzustand zur kosmopolitischen Gesellschaft. Springer Fachmedien Wiesbaden.

Miethe, Ingrid (2016): Paulo Freire: Pedagogy of the Opressed, New York: Herder and Herder 1970, (dt. Pädagogik der Unterdrückten, Stuttgart: Kreuz-Verlag 1971, 218 S.). Hg. v. Salzborn S. Klassiker der Sozialwissenschaften. Wiesbaden: Springer VS.

Neubert, Stefan (2004): Pragmatismus-thematische Vielfalt in Deweys Philosophie und in ihrer heutigen Rezeption. In: Hickman, L. A., Neubert, S., Reich, K., John Dewey (Hg): Zwischen Pragmatismus und Konstruktivismus, Münster: Waxmann, 13-27.

Neuhouser, Frederick (2011): Jean-Jacques Rousseau and the origins of autonomy. In: Inquiry 54(5) (2011), 478-493.

Raya M. J. (2014): Lehrerautonomie: Auf dem Weg zu Freiheit und Eigenverantwortung. In: Jahrbuch Deutsch als Fremdsprache, 40 (2014), 147-162.

Rousseau, Jean Jacques (2011): Emile ya da Eğitim Üzerine (çev. Y. Avunç). İstanbul: Türkiye İş Bankası Kültür Yayınları.

Shala, Erduana (2014): Die Autonomie des Menschen und der Maschine - gegenwärtige Definitionen von Autonomie zwischen philosophischem Hintergrund und technologischer Umsetzbarkeit. Redigierte Version der Magisterarbeit, Karlsruhe KIT: Inst. für Philosophie.

Tassinari, Maria Giovanna (2010): Autonomes Fremdsprachenlernen. Komponenten, Kompetenzen, Strategien. Deutschland: Peter Lang.

Teng, Feng (2019): Autonomy, Agency, and Identity in Teaching and Learning English as a Foreign Language. Singapore: Springer.

Textor, Martin R. (2000): Der konstruktivistische Ansatz. (https://www.kindergartenpaedagogik.de/fachartikel/paedagogische-ansaetze/moderne-paedagogische-ansaetze/145 (letzter Zugriff: 12.11.2020).

Tütüncü, Koray (2013): Raising Democratic and Autonomous Individual: What can we Learn From Jean-Jacques Rousseau's Emile?. In: Ekonomik ve Sosyal Araştırmalar Dergisi, Güz 2013, Cilt:9, Y11:9, Say1: 2, 9, 303-327.

Wilson, Terri S. / Ryg, Matthew A. (2015): Becoming autonomous: Nonideal theory and educational autonomy. In: Educational Theory 65(2), 127-150. 
Wrana, Daniel (2008): Autonomie und Struktur in Selbstlernprozessen. Gesellschaftliche, lerntheoretische und empirische Relationierungen. In: Maier Reinhard, Christiane und Wrana, Daniel (Hg.): Autonomie und Struktur in Selbstlernarchitekturen. Empirische Untersuchung zur Dynamik von Selbstlernprozessen. Opladen: Budrich UniPress, 31-101.

Yazıcl, Şakir Ahmet (2016): The Relationship between the teacher autonomy and learner autonomy support behaviors. In: Journal of Educational Sciences Research, 6(2), 1-23. 